\title{
Beyond physiotherapy and pharmacological treatment for fibromyalgia syndrome: tailored tACS as a new therapeutic tool
}

\author{
Laura Bernardi ${ }^{1} \cdot$ Margherita Bertuccelli $^{1,2}$ (1) Emanuela Formaggio ${ }^{1} \cdot$ Maria Rubega $^{1} \cdot$ Gerardo Bosco $^{3}$. \\ Elena Tenconi ${ }^{4} \cdot$ Manuela Cattelan $^{5} \cdot$ Stefano Masiero $^{1,2} \cdot$ Alessandra Del Felice $^{1,2}$
}

Received: 18 May 2020 / Accepted: 7 November 2020 / Published online: 25 November 2020

(c) The Author(s) 2020

\begin{abstract}
Fibromyalgia syndrome (FMS) is a complex pain disorder, characterized by diffuse pain and cognitive disturbances. Abnormal cortical oscillatory activity may be a promising biomarker, encouraging non-invasive neurostimulation techniques as a treatment. We aimed to modulate abnormal slow cortical oscillations by delivering transcranial alternating current stimulation (tACS) and physiotherapy to reduce pain and cognitive symptoms. This was a double-blinded, randomized, crossover trial conducted between February and September 2018 at the Rehabilitation Unit of a teaching Hospital (NCT03221413). Participants were randomly assigned to tACS or random noise stimulation (RNS), 5 days/week for 2 weeks followed by ad hoc physiotherapy. Clinical and cognitive assessments were performed at $T_{0}$ (baseline), $T_{1}$ (after stimulation), $T_{2}$ (1 month after stimulation). Electroencephalogram (EEG) spectral topographies recorded from 15 participants confirmed slow-rhythm prevalence and provided tACS tailored stimulation parameters and electrode sites. Following tACS, EEG alpha1 ([8-10] Hz) activity increased at $T_{1}(p=0.024)$ compared to RNS, pain symptoms assessed by Visual Analog Scale decreased at $T_{1}\left(T_{1}\right.$ vs $T_{0} p=0.010$ ), self-reported cognitive skills and neuropsychological scores improved both at $T_{1}$ and $T_{2}$ (Patient-Reported Outcomes in Cognitive Impairment, $T_{0}-T_{2}, p=0.024$; Everyday memory questionnaire, $T_{1}$ compared to RNS, $p=0.012$; Montréal Cognitive Assessment, $T_{0}$ vs $T_{1}, p=0.048$ and $T_{0}$ vs $T_{2}, p=0.009$; Trail Making Test B $T_{0}-T_{2}, p=0.034$ ). Psychopathological scales and other neuropsychological scores (Trail Making Test-A; Total Phonemic Fluency; Hopkins Verbal Learning Test-Revised; Rey-Osterrieth Complex Figure) improved both after tACS and RNS but earlier improvements $\left(T_{1}\right)$ were registered only after tACS. These results support tACS coupled with physiotherapy in treating FMS cognitive symptoms, pain and subclinical psychopathology.
\end{abstract}

Keywords Pain $\cdot$ Fibrofog $\cdot$ Non-invasive transcranial stimulation $\cdot$ Rehabilitation $\cdot$ Random noise stimulation (RNS)

Laura Bernardi and Margherita Bertuccelli authors contributed equally to this work.

Electronic supplementary material The online version of this article (https://doi.org/10.1007/s00406-020-01214-y) contains supplementary material, which is available to authorized users.

Margherita Bertuccelli

margherita.bertuccelli@phd.unipd.it

1 Department of Neuroscience, Section of Rehabilitation, University of Padova, via Giustiniani 3, 35128 Paduas, Italy

2 Department of Neurosciencse and Padova Neuroscience Center, University of Padova, 35131 Padua, Italy

3 Department of Biomedical Sciences, University of Padova, Via Marzolo 3, 35031 Padua, Italy

\section{Introduction}

Fibromyalgia syndrome (FMS) is a complex chronic pain disorder, defined as widespread musculoskeletal pain in the absence of demonstrated tissue damage, and associated with symptoms ranging from affective disturbances, fatigue, and sleep alterations, to cognitive dysfunctions [1]. Cognitive difficulties are referred by $50-80 \%$ of people with FMS [2] and are ranked as the fifth most severe symptom [3]. "Fibrofog" was coined to describe the typical subjective experience

4 Department of Neuroscience and Padova Neuroscience Center, Psychiatric Clinic, University of Padova, Via Giustiniani 3, 35128 Padua, Italy

5 Department of Statistical Sciences, University of Padova, via C. Battisti 241, 35121 Padua, Italy 
of cognitive dysfunction in FMS, characterized among other, by concentration difficulties, forgetfulness, mental confusion and inability to multitask [4]. One of the most frequently reported cognitive dysfunctions in FMS is attention deficit [5]: people with FMS show poor performances in cognitive tests requiring to deal with distractors or any source of stimulus competition, such as divided attention, inhibition, set-shifting [6], working memory [7], semantic memory and speed of processing [8]. It is still unclear if these cognitive symptoms are primarily disease manifestation or a consequence of it. Many co-occurring symptoms in FMS such as depression, anxiety, sleep disturbances and pain perception may contribute and account for cognitive problems, even if no study found an unequivocal relation between these factors and the cognitive performance [4]. However, a relationship between pain intensity, affective and cognitive dysfunction has been postulated based on the overlap of brain areas involved in pain processing and cognition [9, 10]. This observation leads to the hypothesis that cognitive alterations in FMS arise because of resource competition with pain processing [6].

Physiotherapy is nowadays the most widely recognized and beneficial treatment for fibromyalgic pain symptoms. Literature reports effective pain and depression reduction associated with aerobic and strengthening exercises [11]. However, physiotherapy alone is not able to provide longlasting effects involving other symptoms than pain.

Current research is focusing on biomarkers which may account for both pain processing alterations and cognitive fibrofog. Single-photon Emission Computed Tomography (SPECT) and Functional Magnetic Resonance Imaging (FMRI) revealed abnormal activation of thalamic nuclei, sensory cortex, anterior cingulate, insula and prefrontal cortices during pain processing in FMS [12, 13]. Electroencephalographic (EEG) studies show increased theta rhythm primarily localized in frontal brain regions and anterior cingulate cortex $[14,15]$, which are part of the thalamo-cortical circuit. On this ground, chronic pain referred by people with FMS has been interpreted as the result of a "thalamo-cortical dysrhythmia" [14] characterized by a shift of oscillatory frequencies in the thalamo-cortical circuits.

Neuromodulation techniques [non-invasive brain stimulation (NIBS): transcranial magnetic (TMS) and transcranial direct current stimulations (tDCS)] may modulate EEG frequency rhythms $[16,17]$ and shift cortical EEG generators $[18,19]$. Up to now, NIBS has been mainly applied in FMS with the hypothesis of reducing the increased activity described over prefrontal and sensory cortices. TDCS was previously administered with this aim. By generating low-intensity sub-threshold electrical fields, tDCS is able to modify neuronal transmembrane potentials and in turn modulate cortical excitability by bringing the underlying neurons closer to their firing threshold $[20,21]$. Repetitive
TMS (rTMS) delivered over M1 was administered as well to reduce FMS pain symptomatology [22]. However, studies on the effect of rTMS and tDCS report a variable efficacy on amelioration of symptoms and quality of life in FMS $[23,24]$.

Focusing instead on abnormal oscillatory activity in FMS, EEG activity normalization may be considered a therapeutic target. No study has explored the effect of transcranial alternating current stimulation (tACS) for the treatment of this clinical population [25].

TACS is a non-invasive, handy technique which may modulate endogenous brain oscillations $[18,25]$ when administrated as an alternate, sinusoid current. It has been demonstrated that tACS is able to shift EEG rhythms in other thalamo-cortical dysrhythmias [26]. Unlike tDCS, tACS does not induce any polarization effect but can modulate the ongoing brain activity by forcing the membrane potential to oscillate away from its resting state towards hyper-polarized or depolarized state. This results in the so-called entrainment effect: increasing of neuronal firing time-locked to the frequency of stimulation [27].

We hypothesize that tACS delivered over the cortical area showing the greatest EEG alteration (i.e., higher slow rhythms power) may have beneficial effects on both pain and cognition by shifting EEG activity towards physiological frequencies. To test this hypothesis, we applied tACS as a primer for a specific rehabilitation program.

\section{Materials and methods}

The randomized, double-blind, crossover design was approved by the ethics Committee of the teaching Hospital of Padova University, Italy, (protocol no. 3507/AO/15). Each participant, before taking part in the experiment, was informed about the study and provided written informed consent. The study was registered on ClinicalTrials.Gov (NCT03221413).

The present study reports preliminary data from participants with chronic pain. The original protocol planned inclusion of individuals with neuropathy; due to the principal investigator change of affiliation, access to this clinical population was no more possible and FMS was included, given that both FMS and neuropathic pain are characterized by increased slow thalamo-cortical oscillations in theta frequency band $[14,16]$. This allowed the application of the same stimulation protocol. Clinical and neuropsychological tests were adjusted and tailored to FMS. To enhance research transparency, we used the recently updated CONSORT guidelines for cross-over trials [28] and the CONSORT checklist for crossover designs (see Supplementary material 1). 
Primary outcome was reduction of the main FMS symptom, pain, measured with a visual analog scale (VAS) on a Likert scale from 0 to 10 .

\section{Participants}

Participants $(N=15,2$ males; age: mean \pm standard deviation: $53.07 \pm 4.18$ years) were recruited as volunteers by the Italian Association of FMS (AISF) and enrolled by a blinded researcher in charge of the study conduction (LB), who also assigned them to sequence of intervention. Inclusion criteria were: diagnosis according to the diagnostic criteria for fibromyalgic syndrome [29]; score higher than 3 at the Visual Analog Scale for pain (VAS); pain non-responsive to at least two analgesic drugs administered in adequate dose for at least 3 months; stable pharmacological treatment during the study. Exclusion criteria were: contraindications to neurostimulation (pregnancy, metal fragments/implants, epilepsy, previous skull fractures, pacemaker); comorbid psychiatric illnesses or substance abuse disorders; minor age. Scalp EEG control data from twenty-one healthy volunteers ( 9 males; age: $45.14 \pm 14$ years) were obtained [30] using the same EEG setting.

\section{EEG data acquisition and analysis}

Five minutes of open-eyes resting-state EEG signal (32-channel system; BrainAmp 32MRplus, BrainProducts $\mathrm{GmbH}$, Munich, Germany) were acquired at a sampling rate of $5 \mathrm{kHz}$ with the reference between $\mathrm{Fz} / \mathrm{Cz}$ and ground anterior to Fz positioned according to a 10/10 system, band-pass filtered at $0.1-1000 \mathrm{~Hz}$ and digitized.

The data were processed in Matlab (MathWorks, Natick, MA) using personalized scripts based on EEGLAB toolbox
[31]. The EEG recordings were band-pass filtered from 1 to $30 \mathrm{~Hz}$ and down-sampled at $500 \mathrm{~Hz}$. Visible artifacts (eyes movements, cardiac activity, and scalp muscle contraction) were removed using independent component analysis, and data were processed with a common average reference. Two-seconds EEG epochs (i.e., non-overlapping segments of 1000 samples) were extracted for each participant and a fast Fourier transform (FFT) was applied. The recordings were Hamming windowed to control for spectral leakage. Power spectral density $\left(\mu \mathrm{V}^{2} / \mathrm{Hz}\right)$ was estimated for all frequencies and the relative power (\%) was computed by dividing the power of each frequency band (delta [1-4 Hz], theta [4.5-7.5 Hz], alpha1 [8-10 Hz], alpha2 [10.5-12.5 Hz], beta [13-30 Hz]) with the total power in the range $1-30 \mathrm{~Hz}$.

$\mathrm{A} z$ test $(p<0.05)$ was used to compare each participant versus controls and a statistical map, defining the electrodes in which relative power value differs from those of the control group, was provided [32].

\section{Trial design, stimulation parameters and tACS treatment}

Participants were randomly assigned with a computergenerated list (allocation ratio 1:1) to tACS or random noise stimulation (RNS as active sham). Each participant underwent 10 stimulation sessions lasting $30 \mathrm{~min}, 5$ times a week, for two consecutive weeks, followed by $60 \mathrm{~min}$ of physical rehabilitative exercise. After a wash-out interval of 4 weeks from the conclusion of the first cycle, participants were crossed to the other group (Fig. 1). Participants were tested before program start $\left(T_{0}\right)$, at conclusion of each cycle [stimulation and physical rehabilitation $\left(T_{1}\right.$; $\left.T_{1}{ }^{\prime}\right)$ ], and after the 4-week wash-out interval $\left(T_{2} ; T_{2}{ }^{\prime}\right)$, with resting EEG, VAS, SF36, neuropsychological tests

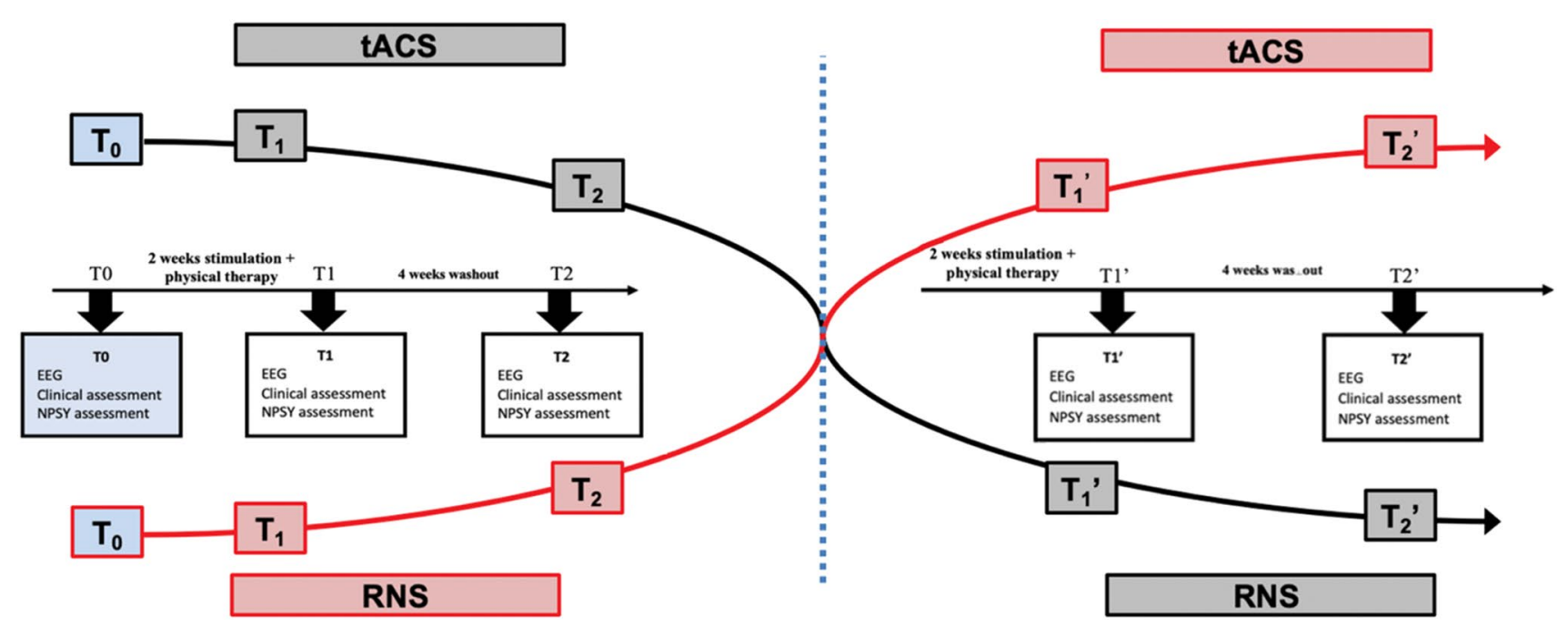

Fig. 1 Schematic representation of the experimental design 
and questionnaires. $T_{0}$ values were recorded only before the start of the whole experiment.

Baseline (before the whole protocol started) restingstate cerebral activity provided stimulation parameters (frequency and anode position on the scalp). EEG from participants was compared with those of a healthy control group to identify scalp areas in which a significant difference in frequency band spectral power was detected. We supported the hypothesis that a non-invasive stimulation though potentially different from the natural oscillations of the target brain region could modulate neuronal networks and shift oscillations into a physiological range. For this reason, we compared the power spectra of the participants with the one of the controls. Each participant was stimulated in the EEG frequency band that presented lower amplitudes compared to controls. Those showing higher slow frequencies (theta, delta, alpha1) spectral power were stimulated with beta-tACS at $30 \mathrm{~Hz}$, while the ones showing higher fast frequencies (beta, alpha2) were stimulated with theta-tACS at $4 \mathrm{~Hz}$. Figure 2 displays statistical maps derived from one participant showing higher theta activity over left motor area, compared to controls, and thus stimulated with beta-tACS over that cortical area. In case a subgroup should display a prevalence of faster frequencies (alpha2, beta), they were to be stimulated with slow tACS at $4 \mathrm{~Hz}$.

Stimulation was applied by a battery driven external stimulator (BrainStim, E.M.S., Bologna, Italy) via two sponge electrodes $(5 \times 7 \mathrm{~cm})$, with an intensity ranging from 1 to $2 \mathrm{~mA}$. Anode was positioned for each subject over the scalp area showing highest power spectral difference; cathode over the ipsilateral mastoid. The RNS was an alternate current with random amplitude and frequency, respectively, in the intervals (1-2) $\mathrm{mA}$ and $(0-100) \mathrm{Hz}$, with electrodes applied over the same sites as for real stimulation". [17].

\section{Clinical assessment}

The following clinical scales were administered to test pain and the self-reported health state:

(1) Visual Analog Scale (VAS) [33]: a 10-point Likert scale, ranging from none to extreme amount of pain.

(2) Short Form 36-item Health Survey (SF-36) [34]: the short form of a questionnaire inquiring participants' health state. The 36 items are clustered into 8 domains: physical activity/functionality (10 items), limitations due to physical health (4 items), limitations due to emotional status ( 3 items), physic pain ( 2 items), general healthy sate perception ( 5 items), energy/fatigue (4 items), social activities ( 2 items), mental health (5 items) and one single question on the perceived changes in the health state.

\section{Neuropsychological and psychopathological assessment}

To test cognitive, affective and psychopathological domains, we designed a battery which comprises the following standardized neuropsychological tests and questionnaires for:

(1) Psychopathological self-assessment: Beck Depression Inventory-II (BDI-II) [35]; Brief Symptom Inventory (BSI) [36]; the State-Trait Anxiety Inventory [37].

(2) Self-reported cognitive assessment: Patient-Reported Outcomes in Cognitive Impairment (PROCOG-P) [38]; Everyday Memory Questionnaire Revised (EMQ-R) [39].

(3) Neuropsychological assessment: the Montréal Cognitive Assessment (MoCA) [40]; the Rey-Osterrieth Complex Figure Test [41]; the Digit Symbol-Coding (from the Wechsler Adult Intelligence Scale 4th edition [42]; the Hopkins Verbal Learning Test-Revised [43];

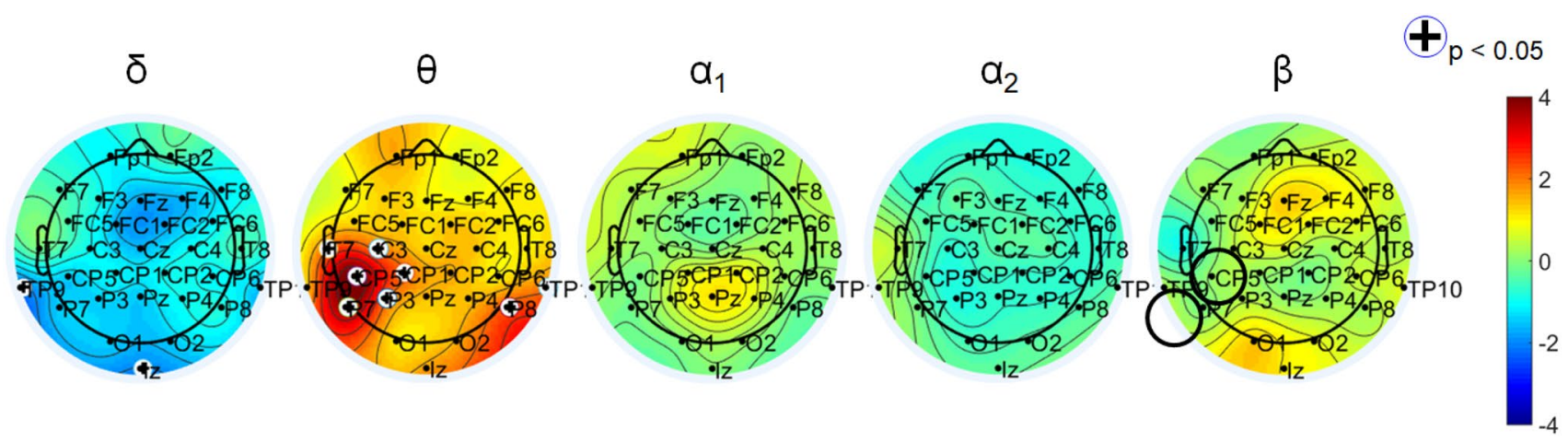

Fig. 2 Statistical maps ( $z$ values) derived from one subject with FMS vs. control group. Participant n. 6 shows higher theta activity, compared to controls, over left motor area. She was stimulated with beta
tACS over CP5 (black circles: anode over area of highest theta power, cathode over ipsilateral mastoid) 
the Trail Making Tests A and B [44]; the Phonemic Verbal Fluency task [45]. For detailed description of each test, see Appendix A.

\section{Physiotherapy program}

The European League Against Rheumatism (EULAR) guidelines have been used to design the physiotherapy program [46]. Physiotherapy program included $30 \mathrm{~min}$ of aerobic exercise followed by $15 \mathrm{~min}$ of muscular stretching and 15 min of breathing and guided relaxation techniques. Participants received 60-min physical rehabilitation at the end of each stimulation session, 5 times a week, for two consecutive weeks, both in the first and second experimental arms. See the online resources (Table T1) for the complete physiotherapy program.

\section{Statistical analysis}

Region of interests (ROIs) were identified based on electrodes location: right frontal ROI: Fp2, F8, F4; right motor ROI: FC2, FC6, C4, Cp2; right parietal ROI: CP6, P8, P4. Contralateral ROIs were identified on the left hemisphere. EEG frequency power was calculated for each of these ROIs and used for subsequent analysis. Statistical analyses aimed at detecting differences in EEG frequencies, neuropsychological, psychopathological or clinical variables with respect to baseline and intervention. Each comparison was based on complete case analysis, so all available subjects were considered when their observations could be collected. Nonparametric methods were employed due to sample size. Spectral power of all EEG bands over different ROIs were compared to detect differences between the conclusion of the cycle $\left(T_{1}\right)$ and the end of the wash-out interval $\left(T_{2}\right)$ and between the condition of the subjects in each of those times $\left(T_{1}\right.$ and $\left.T_{2}\right)$ and their baseline $\left(T_{0}\right)$ by means of the Wilcoxon signed-rank test for paired samples. EEG frequencies were compared also between tACS and RNS with a paired samples Wilcoxon signed-rank test. All changes in variables related to the neuropsychological and psychopathological assessments and to the clinical assessment collected at different time point were tested with the same scheme with a Wilcoxon signed-rank test, assuming the usual critical level 0.05 . All statistical analyses were performed with the R statistical software [47].

\section{Test power calculation}

Sample size was computed on the basis of a functional outcome which is not available for subjects who actually entered the study. For this reason, an analysis of the power of the test employed was performed. The primary outcome is the change in the VAS scale between the beginning of the study and the end of intervention. Since the Wilcoxon ranksum test is a non-parametric test, the assumption of a standard parametric distribution for the data under the alternative hypothesis is avoided. A discrete distribution is assumed for the difference in VAS which ranges between -1 and 6, with probabilities equal to those empirically observed, thus the median difference equals 1.5. Under such assumptions, a simulation study was performed in order to detect the power of the Wilcoxon signed-rank test. Based on 10,000 simulations, with the observed sample size, the power of the test is $85 \%$.

\section{Results}

Twenty-four participants were recruited. Enrolment flow diagram is reported in the online resources (Fig. F1). For demographic characteristics see Table 1. Seven participants did not meet inclusion criteria ( 2 had contraindication to tACS, 3 had a psychiatric disease, 2 had recently changed drug regimen). Of the remaining 17, 15 completed the first arm of the study (1 drop out, 1 change of drugs during trials), and 11 both study arms ( 1 change of drugs, 1 due to stroke, 2 dropouts for personal reasons).

\section{EEG}

The open-eyes resting-state EEG confirmed low rhythm prevalence over fronto-central cortical regions (11/15 participants) $[13,15]$. Alpha 1 power increased at $T_{1}(p=0.024$, CI $(-1.89,-0.13))$ after beta-tACS compared to RNS over bilateral M1 (Fig. 3). Alpha 2 showed a non-significant increase between $T_{1}$ and $T_{2}$ both after tACS and RNS over bilateral M1.

\section{Clinical assessment scales}

VAS scores significantly decreased in 9 cases out of 14 from $T_{0}$ to $T_{1}(p 0.010, \mathrm{CI}(0.5,4.0))$ after tACS. This effect was no longer seen at $T_{2}$, where just 4 subjects out of 14 reported a reduced pain perception compared to $T_{1}$.

After RNS, no significant improvement was seen between $T_{0}$ and $T_{1}$ as just in 7 cases out of 14 we observed a reduction in VAS scores $(p=0.062)$ as well as between $T_{1}$ and $T_{2}$ where just 4 subjects reported pain symptoms reduction $(p=0.757)$.

Several items of the Short Form 36-item Health Survey (SF-36) improved after both tACS and RNS. See the online resources (Tables T2, T4) for significant p-values and median scores related to each subitem. 
Table 1 Demographic and clinical characteristics of included participants

\begin{tabular}{|c|c|c|c|c|c|c|c|}
\hline Subject & Age (years) & Sex & Education (years) & Prevailing rhythm & Stimulation site & Stimulation frequency & Pharmakon \\
\hline 1 & 51 & $\mathrm{~F}$ & 16 & Theta & $\mathrm{F} 3$ & $30 \mathrm{~Hz}$ & Duloxetine $(60 \mathrm{mg})$ \\
\hline 2 & 49 & $\mathrm{~F}$ & 12 & Theta & $\mathrm{C} 3$ & $30 \mathrm{~Hz}$ & Amitriptyline (10 mg ttx2) \\
\hline 3 & 51 & $\mathrm{~F}$ & 13 & Theta & $\mathrm{Cp} 5$ & $30 \mathrm{~Hz}$ & $\begin{array}{l}\text { Hydroxychloroquine } \\
(200 \mathrm{mg})\end{array}$ \\
\hline 4 & 56 & $\mathrm{~F}$ & 13 & Alpha 1 & $\mathrm{C} 4 / \mathrm{Cp} 2$ & $30 \mathrm{~Hz}$ & Tizanidine (4 mg) \\
\hline 5 & 55 & $\mathrm{~F}$ & 16 & Theta & $\mathrm{C} 3 / \mathrm{Cp} 5$ & $30 \mathrm{~Hz}$ & Venlafaxine (75 mg) \\
\hline 6 & 49 & $\mathrm{~F}$ & 9 & Theta & $\mathrm{Cz}-\mathrm{Fz}$ & $30 \mathrm{~Hz}$ & $\begin{array}{r}\text { Gabapentin }(300 \times 3), \\
\text { venlafaxine }(75 \mathrm{mg})\end{array}$ \\
\hline 7 & 55 & $\mathrm{~F}$ & 8 & Theta & $\mathrm{Cp} 5$ & $30 \mathrm{~Hz}$ & $\begin{array}{c}\text { Alprazolam }(0,25 \mathrm{mgx} 2), \\
\text { pregabalin }(150 \mathrm{mgx} 3), \\
\text { Trzodone }(100 \mathrm{mg})\end{array}$ \\
\hline 8 & 50 & M & 11 & Alpha 2 & $\mathrm{Cz}-\mathrm{Pz}$ & $4 \mathrm{~Hz}$ & $\begin{array}{l}\text { Alprazolam }(25 \mathrm{mgx} 2), \\
\text { Amitriptyline }(10 \mathrm{mg}), \\
\text { Tizanidine }(2 \mathrm{mgx} 2)\end{array}$ \\
\hline 9 & 50 & $\mathrm{~F}$ & 13 & Alpha 2 & $\mathrm{Pz}$ & $4 \mathrm{~Hz}$ & $\begin{array}{l}\text { Gabapentin }(300 \times 2), \\
\text { Duloxetine }(60 \mathrm{mg}), \\
\text { Lormetazepam }(2 \mathrm{mg}), \\
\text { Zolpidem }(50 \mathrm{mg}), \text { Sirda- } \\
\text { lud }(2 \mathrm{mg}), \text { Tapendatol } \\
(50 \mathrm{mgx} 2)\end{array}$ \\
\hline 10 & 50 & $\mathrm{~F}$ & 16 & Theta & F4 & $30 \mathrm{~Hz}$ & $\begin{array}{l}\text { Pregabalin }(100 \mathrm{mg}), \\
\text { Duloxetine } 60 \mathrm{mg}\end{array}$ \\
\hline 11 & 65 & $\mathrm{~F}$ & 9 & Delta & C3 & $30 \mathrm{~Hz}$ & - \\
\hline 12 & 53 & $\mathrm{~F}$ & 11 & Theta & Cp5 & $30 \mathrm{~Hz}$ & - \\
\hline 13 & 57 & $\mathrm{~F}$ & 13 & Theta & $\mathrm{Cp} 5$ & $30 \mathrm{~Hz}$ & $\begin{array}{l}\text { Duloxetine }(60 \mathrm{mg}) \text {; Prega- } \\
\text { balin }(75 \mathrm{mg})\end{array}$ \\
\hline 14 & 52 & $\mathrm{~F}$ & 8 & Beta & C3 & $4 \mathrm{~Hz}$ & - \\
\hline 15 & 53 & $\mathrm{M}$ & 17 & Beta & $\mathrm{Pz}$ & $4 \mathrm{~Hz}$ & - \\
\hline
\end{tabular}

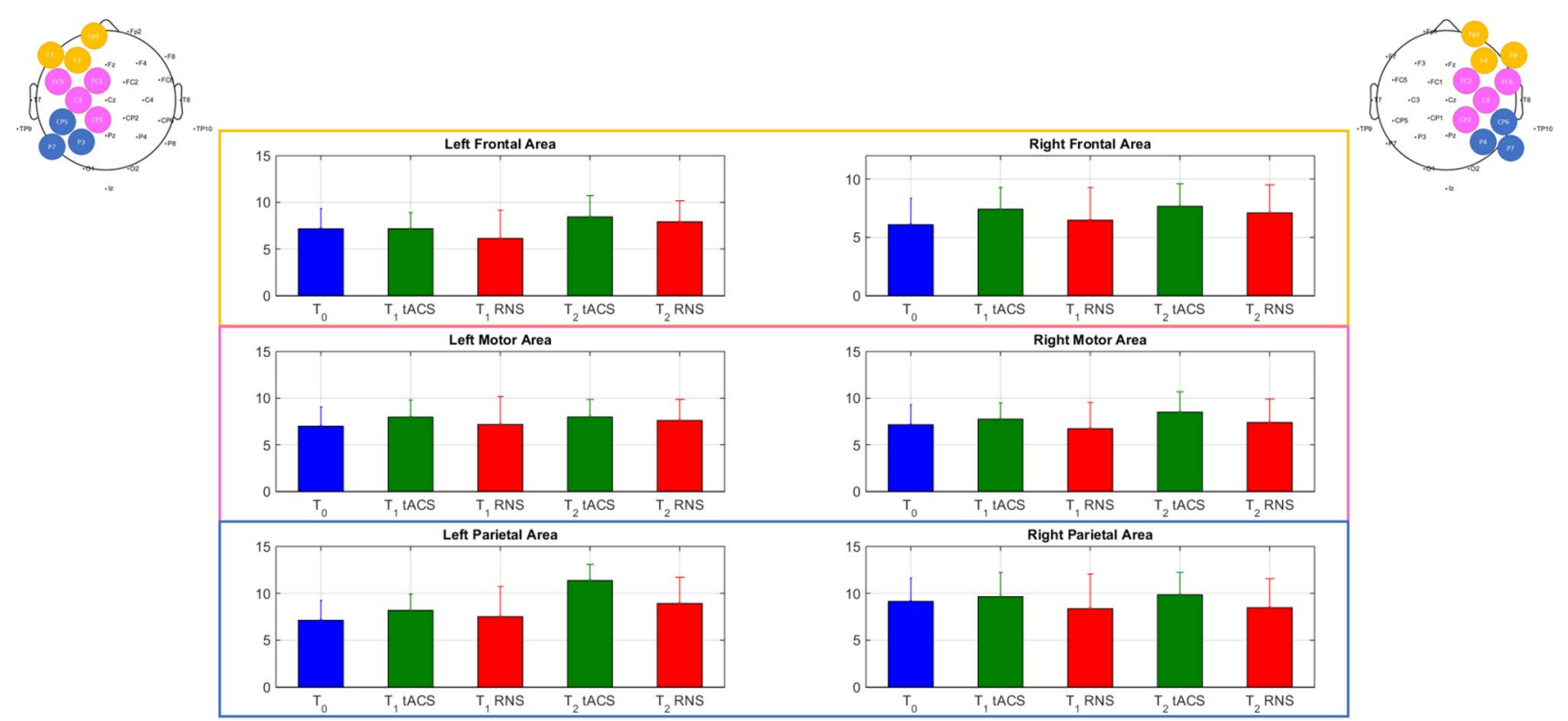

Fig. 3 Group results. Median relative power and standard error in alpha1 range at each evaluation time point $\left(T_{0}, T_{1}\right.$ tACS, $T_{1}$ RNS, $T_{2}$ tACS, $T_{2}$ RNS). Region of interests are identified based on electrodes location (mean of relative power): left frontal area -i.e., Fp1, F7, F3; right frontal area-i.e., Fp2, F8, F4; Left motor area-i.e., FC1, FC5, C3, Cp1; right Motor Area-i.e., FC2, FC6, C4, Cp2; left Parietal Area-i.e., CP5, P7, P3; Right parietal area-i.e., CP6, P8, P4 


\section{Neuropsychological and psychopathological assessment}

(1) Psychopathological self-assessment: the State-Trait Anxiety Inventory, assessing baseline state and trait anxiety levels, shows low trait anxiety in our sample at baseline (mean \pm standard deviation $=37.26 \pm 7.63$; median $=38$ ). Depressive symptoms, assessed with the BDI-II, decreased in the tACS group between $T_{0}$ and $T_{1}(p<0.001, \mathrm{CI}(4.0,12.0))$, and $T_{0}$ and $T_{2}(p=0.006$, CI $(5.0,13.0))$, as well as in the RNS group between $T_{0}$ and $T_{1}(p=0.008, \mathrm{CI}(1.0,10.5))$. The BSI global severity index score, a measure of the current level of perceived symptomatology, significantly decreased after both tACS and RNS between $T_{0}$ and $T_{1}$ (respectively, $p=0.042$, CI $(0.01,0.11)$, and $p=0.027$, CI $(0.02$, $0.23)$ ). Several subitems of the BSI improved after both tACS and RNS. Tables T3 and T5 in the online resources, respectively, report the p-values and the median scores for each subitem.

(2) Self-reported cognitive assessment: the skill loss subitem of the Patient-Reported Outcomes in Cognitive Impairment (PROCOG-P) improved after tACS at $T_{0}-T_{2}(p=0.024$, CI $(0.05,0.59))$. The performance in the Everyday memory questionnaire (EMR.Q) resulted significantly improved in the group receiving the tACS compared to the RNS at $T_{1}(p=0.012, \mathrm{CI}(1.5,16.5))$.

(3) Neuropsychological assessment: among neuropsychological tests, a significant improvement is observed in the MoCa scores after tACS at $T_{0}-T_{1}(p=0.048$, $\mathrm{CI}(-4.5,0.0))$ and at $T_{0}-T_{2}(p=0.009, \mathrm{CI}(-4.5$, $-0.5)$ ). In the Trail Making Test A (TMT-A), we observed a significant increased speed in the RNS group between $T_{0}$ and $T_{2}(p=0.035, \mathrm{CI}(0.0,15.0))$. The TMT-B time significantly decreased in the tACS group between $T_{0}$ and $T_{2}(p=0.034, \mathrm{CI}(0.0,37.5))$. Total Phonemic Fluency scores increased in the group receiving tACS between $T_{0}$ and $T_{2}(p=0.013, \mathrm{CI}(-10.0,-1.0))$ and errors decreased $(p=0.025, \mathrm{CI}(0.0,2.0))$. In the RNS group, an increase was observed between $T_{1}$ and $T_{2}(p=0.006$, CI $(-9.0,-1.5))$. In the HVLT-R, the tACS group improved significantly both at $T_{0}-T_{2}$ and $T_{1}-T_{2}$ (respectively, $p=0.008$, CI $(-9.0,-1.0)$, and $p=0.009, \mathrm{CI}(-5.5,-1.0))$; the RNS group improved between $T_{0}$ and $T_{2}(p=0.012, \mathrm{CI}(-14.5,-2.0))$. The Rey-Osterrieth Complex Figure time score improved in the tACS group between $T_{0}$ and $T_{1}(p=0.025, \mathrm{CI}$ $(7.0,49.0))$, and $T_{0}$ and $T_{2}(p=0.049$, CI $(0.0,42.0))$, and between $T_{0}$ and $T_{2}$ after RNS ( $p=0.037$, CI (3.5, 69.0)).

In the online resources (Table T6) are reported the median scores of each administered test.

\section{Discussion}

Our data confirm cognitive and EEG activity abnormalities in a sample of people with FMS. This neurophysiological finding informed the choice of the neurostimulation paradigm: tACS combined with an ad hoc physical program was effective in shifting EEG frequencies, reducing pain, and improving neuropsychological and psychopathological tests.

Slow rhythm prevalence in fronto-central cortices are a hallmark of FMS [16]: fast tACS aimed to interact with these abnormal brain oscillations and shift them towards more physiological frequencies [18, 25]. Eleven out of fifteen participants of the initial sample showed theta rhythm prevalence in frontal regions and/or sensorimotor areas. As hypothesized, tailored tACS normalized EEG activity [48]. Although beta-tACS decreases the prevalent theta power in our sample, the induced shift was towards slightly faster (alpha1 or alpha2) bands and not towards beta band. This observation may be explained with the complexity of the targeted pain circuit, the pain matrix [49]. Neuroimaging and neurophysiological studies demonstrated that nociceptive stimulations activate large brain network comprising somatosensory, insular, cingulate areas, and with a temporal delay frontal and parietal areas [50]. FMS pain-related symptomatology is believed to be associated with neuroplastic changes in this network [51]. Taking this into account, we argue that focal tACS stimulation is less likely to radically impact on a large cortical/subcortical network, like the one represented by the pain matrix. Indeed, tACS efficacy in EEG activity normalization was observed in the treatment of other thalamo-cortical dysrhythmias such as in Parkinson's disease, in which the closely circumscribed thalamo-corticobasal circuit was targeted [18].

We found the primary motor cortex (M1) and sensorimotor areas to emerge as the main targets for the neurostimulation based on the topography of EEG abnormalities. Although M1 is not directly part of the pain matrix, previous studies proved its modulatory role in other chronic pain syndromes [51]. M1 stimulation appears to activate phasic and rapid activity of lateral thalamic nuclei, which in turn activate a cascade of events in the medial thalamus, anterior cingulate/orbitofrontal cortex and periaqueductal gray matter.

Thus, we argue that tACS delivered over M1/sensorimotor cortices may have a modulatory effect on the pain matrix and consequently reduce pain, as proved by reduced VAS score. The absence of significant improvements at $T_{2}$ confirms previous reports $[52,53]$ on the lack of long-lasting effects of NIBS: our experimental paradigm was able to modulate pain during the experiment itself and immediately after, but the pain reduction faded after 4 weeks. This observation calls for the development of portable NIBS devices which may be used for home therapy. In addition, the lack 
of effects after RNS can be considered a proof as well of the benefit induced by the real stimulation and physiotherapy treatment.

Physiotherapy may be a co-factor in pain reduction; however, as only the tACS group reported a significant decrease of VAS, an effect of stimulation per se accounting for this outcome is highly likely. TACS may have acted as a primer for the motor cortex, increasing the potential excitability of the underlying cortex and amplifying the ensuing physiological activation obtained through motion.

While the results of tACS on pain suggest a determinant role of neurostimulation, the overall positive effect of physical activity on health perception is well known and observed also in our cohort.

A general health increased is observed in both groups (tACS and RNS), as assessed by SF-36 scores of functionality, energy, social activity and pain subitems.

Similraly, physical activity may have affected also BSI and BDI-II. BSI assesses somatization, obsessive compulsive tendencies and anxiety; scores improved in both groups between $T_{0}$ and $T_{1}$. BDI-II assesses depressive symptoms; it decreased significantly in short and medium term $\left(T_{0}-T_{1}\right.$ and $T_{1}-T_{2}$ ) in the tACS group. It is unclear if this improvement results from a primary effect of the stimulation and the physiotherapy, both previously demonstrated to be effective in depressive symptoms amelioration $[54,55]$ or if it is a consequence of pain reduction per se. Pain and depression are strictly linked; thus, a reduction of one can lead to beneficial effects on the other. However, depressive symptoms seem to ameliorate also in the RNS group in the short term $\left(T_{1}\right)$, which did not show a significant reduction in VAS scores. In this case, the positive effect of physiotherapy may have played a determinant role.

The PROCOG-P skill loss subitem as well as the EMR.Q improvement in the tACS group can be interpreted as a consequence of the stimulation, which by increasing alpha 1 band prevalence, may have boost cognitive performances and the relative perception of self-cognitive abilities. However, pain and depression reduction may have played a role by influencing subjects' general attitude on their capacities. This underlies the need to test cognitive abilities also with neuropsychological standardized test, to disentangle subjective and objective measures of cognitive functioning.

A positive correlation between theta power increase and cognitive deficit in healthy adults is reported [56]; whereas, high power of alpha rhythm is positively correlated with memory and attention performances [57]. TACS stimulation, increasing alpha1 band prevalence, may explain the observed improvements in the MoCA scores, which comprises attention, short-term memory and working memory tasks.
The same can be assumed for TMT-B performance assessing divided attention and set-shifting, whose improvement is observed only in tACS condition. On the contrary, TMT-A performance speeds up only in the RNS group. We argue that this task, assessing mainly visual searching abilities, could have been less influenced by the protocol as less involved in FMS symptomatology compared to the other domains tested.

The other cognitive tasks comprising the phonemic fluency test, the HVLT-R and the time copy component of the Rey Complex Figure test, improved both after tACS and RNS stimulation. Faster improvement in performances were observed at $T_{1}$ after tACS, compared to the RNS later improvement $\left(T_{2}\right)$. We argue that tACS combined with physical activity is likely to speed up the process of cognitive performance improvement in FMS, which can anyway be triggered by physical rehabilitation. Indeed, many studies highlight the modulation effect of physical activity on cognitive functioning and general wellbeing [58].

By delivering tailored tACS associated with ad hoc rehabilitative intervention, we succeeded in reducing the main concerns reported by people suffering FMS [59]: pain symptoms and cognitive dysfunctions, including both selfreported measures of perceived impairment and neuropsychological tests performance.

The improvements observed in the group receiving RNS combined with physiotherapy can be explained in light of the multiple-level beneficial impact of physical activity on clinical and cognitive symptoms. However, tACS group showed more pervasive and faster symptoms reduction, pointing out stimulation efficacy.

\section{Limitations}

The main limitation is the small sample size. Nevertheless, the sample homogeneity concerning age, sex and education level, adds value to results reliability.

It is pointed out that, considering the dimension of the sample size and the exploratory nature of the study, no corrections for multiple comparisons were performed in hypothesis testing. Future studies should replicate the validity of this treatment approach on a larger sample.

A potential bias could be concomitant drug therapy. Evidence to date suggests interaction effects between drugs with psychotropic effects and neurostimulation techniques [60] Even if not such interaction is clearly reported for tACS, we controlled for possible confounding effects by keeping participants' pharmacological therapy unchanged during the whole protocol. 


\section{Conclusion}

These data provide evidence of the efficacy of combining personalized tACS and physiotherapy in the treatment of pain, cognitive symptoms and subclinical psychopathology of FMS. Even if the involved mechanisms are still not completely understood, tACS over the sensorimotor cortex coupled with physiotherapy seem to be a promising approach in treating this complex syndrome.

Funding Open access funding provided by Università degli Studi di Padova within the CRUI-CARE Agreement. This research did not receive any specific Grant from funding agencies in the public, commercial, or not-for-profit sectors.

\section{Compliance with ethical standards}

Conflict of interest The authors declare that they have no conflict of interest.

Ethics approval This study was performed in line with the principles of the Declaration of Helsinki. Approval was granted by the ethics committee of the teaching Hospital of Padova University, Italy, (Protocol No. 3507/AO/157.

Informed consent A written informed consent was obtained from all participants.

Open Access This article is licensed under a Creative Commons Attribution 4.0 International License, which permits use, sharing, adaptation, distribution and reproduction in any medium or format, as long as you give appropriate credit to the original author(s) and the source, provide a link to the Creative Commons licence, and indicate if changes were made. The images or other third party material in this article are included in the article's Creative Commons licence, unless indicated otherwise in a credit line to the material. If material is not included in the article's Creative Commons licence and your intended use is not permitted by statutory regulation or exceeds the permitted use, you will need to obtain permission directly from the copyright holder. To view a copy of this licence, visit http://creativecommons.org/licenses/by/4.0/.

\section{Appendix A. Neuropsychological testing}

\section{Clinical and Psychopathological assessment}

The beck depression inventory-II (BDI-II) [35] was used to assess depression. It consists of a 21-item questionnaire yielding a composite score of self-reported symptom severity. Standard cut-off scores are: $0-9=$ minimal depression, $10-18=$ mild depression, $19-29=$ moderate depression, and $30-63=$ severe depression.

Brief symptom inventory (BSI) [36] is a self-reported questionnaire designed to identify relevant psychopathological symptoms experienced during the last week. The questionnaire is composed by 53 items concerning nine symptom dimensions: Somatization, Obsession-Compulsion,
Interpersonal Sensitivity, Depression, Anxiety, Hostility, Phobic Anxiety, Paranoid ideation, and Psychoticism. The questionnaire provides also three global indices of distress: Global Severity Index (a measure of current level of symptomatology), Positive Symptom Distress Index (a measure of the intensities of symptoms), and Positive Symptom Total (a measure of total number of experienced symptoms). GSI T scores $>/=63$ are considered clinically significant, also all cases in which two of the subscales scores are 63 or greater.

The state-trait anxiety inventory [37] is a 40-item selfreport questionnaire assessing state and trait anxiety levels. Scores range from 20 to 80 , with higher scores indicating higher levels of anxiety severity.

\section{Cognitive self-assessment}

Patient-reported outcomes in cognitive impairment (PROCOG-P) [38] is a 55-item self-administered questionnaire designed to measure a range of patient-reported symptoms and their impact in patient's daily life during the last two weeks. The instrument is designed to detect the patient's perspective on his/her cognitive impairment and impact. Items are rated on a five-point Likert scale and the questionnaire includes seven subscales whose scoring is calculated as the mean value of items belonging to each specific subscale (range 0-4).

The PROCOG-P subscales are: affect, skill loss, semantic memory, short-term memory, cognitive functioning, long-term memory and social impact. The subscales give extensive description of the patient experience of his/her cognitive impairment and allow us to assess separately different memory-related symptoms and the emotional impact of symptoms and repetitive behaviors. A total score is the sum of all items (range 0-220). Higher scores indicate both greater impact and severity of cognitive impairment.

Everyday memory questionnaire revised (EMQ-R) [39] is a subjective measure of memory deficiency in everyday life during last month. Total score is the sum of all 13 items, ranged from 0 to 41 , with a mean total of 9.75 (SD 8.6), and it is considered as a good measure of change. Higher the score, worse subjective memory functioning.

\section{Neuropsychological assessment}

The montréal cognitive assessment (MoCA) [61] is a 30-point brief cognitive screening scale with short time of administration. MoCA is one of the most commonly used tools in clinics assessing general cognitive functioning. It measures a broad spectrum of cognitive abilities that are relevant to several disorders involving CNS [61]. In particular, MoCA was developed to explore frontal cognitive domains (i.e., attention, executive functions, and conceptual 
thinking), all domains usually already affected in the early stages of these disorders. Authors identified scores less than 26 as a good cut-off for detecting cognitive impairment.

The Rey-Osterrieth complex figure test [41] is a measure of visuo-spatial constructional abilities and visuo-graphic memory, but also cognitive planning, organizational strategies and executive functions. The task is composed of two parts, direct copying (assessing perception and visuo-spatial construction) and delayed reproduction (assessing implicit visuo-spatial memory). We used the three minutes (short) delay to assess visual memory. Given that repeated administrations of the ROCF resulted in significant improvements of performance, we used alternative forms: The Modified Taylor Complex Figure, and two out of the four complex figures devised for repeated assessments by the Medical College of Georgia Neurology group [62].

The digit symbol-coding (from the Wechsler Adult Intelligence Scale 4th edition [42]), is a measure of graphomotor working memory and speed of processing. It consists of digit-symbol pairs followed by a list of digits. Participants must write the corresponding symbol under each digit (ranged from one to nine) as fast as possible in a limited time interval $\left(120^{\prime \prime}\right)$. Digit Symbol appears to be relatively unaffected by intelligence, memory, or learning. Motor persistence, sustained attention, response speed, visuomotor coordination, all have some role in digit symbol performance, which is also affected by education, gender and age. No practice effects appeared after repeated administering [62].

The hopkins verbal learning test-revised [43] is a test that assesses verbal learning and memory. The test consists of three trials of free recall of a 12-item composed of four words belonging to three different semantic categories. The authors provide six parallel forms leading to equivalent results in the normal population. We used three lists $(\mathrm{N} 1$, 5 , and 6) and considered only the free recall as outcome measure.

The trail making tests A and B [44] measure attentional speed, sequencing, visual search and mental flexibility. Part A (TMT-A) assesses motor speed, part B (TMT-B) assesses complex divided attention and set-shifting, difference between B and A (i.e., B/A ratio) gives a measure of cognitive shifting cost and allows us to control for motor impairment. Practice effect is under discussion, especially in the case of short time interval, and we used three parallel forms [63].

The phonemic verbal fluency task [45] requires patients to freely generate as many words as possible that begin with a specific letter (phonemes) in $60 \mathrm{~s}$. The task requires patients to retrieve words of their language and to access their verbal lexicon, focus on the task, select only words following specific rules and avoid repetitions and words that start with phonemes close to the target one. It is therefore considered dependent on executive control, beyond the involvement of verbal abilities. The outcome consists in the total correct words produced through three letters. In the literature different letter combinations are available to longitudinal studies.

\section{References}

1. Sluka KA, Clauw DJ (2016) Neurobiology of fibromyalgia and chronic widespread pain. Neuroscience 338:114-129. https://doi. org/10.1016/j.neuroscience.2016.06.006

2. Bertolucci PHF, De Oliveira FF (2013) Cognitive impairment in fibromyalgia. Curr Pain Headache Rep. https://doi.org/10.1007/ s11916-013-0344-9

3. Mease PJ et al (2008) Identifying the clinical domains of fibromyalgia: contributions from clinician and patient delphi exercises. Arthritis Care Res 59(7):952-960. https://doi.org/10.1002/ art. 23826

4. Kravitz HM, Katz RS (2015) Fibrofog and fibromyalgia: a narrative review and implications for clinical practice. Rheumatol Int 35(7):1115-1125. https://doi.org/10.1007/s00296-014-3208-7

5. Borchers AT, Gershwin ME (2015) Fibromyalgia: a critical and comprehensive review. Clin Rev Allergy Immunol 49(2):100-151. https://doi.org/10.1007/s12016-015-8509-4

6. Glass JM et al (2011) Executive function in chronic pain patients and healthy controls: different cortical activation during response inhibition in fibromyalgia. J Pain 12(12):1219-1229. https://doi. org/10.1016/j.jpain.2011.06.007

7. Glass JM (2009) Review of cognitive dysfunction in fibromyalgia: a convergence on working memory and attentional control impairments. Rheum Dis Clin N Am 35(2):299-311. https://doi. org/10.1016/j.rdc.2009.06.002

8. Bar-On Kalfon T, Gal G, Shorer R, Ablin JN (2016) Cognitive functioning in fibromyalgia: the central role of effort. J Psychosom Res 87:30-36. https://doi.org/10.1016/j.jpsychores.2016.06.004

9. Cook DB, Lange G, Ciccone DS, Liu WC, Steffener J, Natelson $\mathrm{BH}$ (2004) Functional imaging of pain in patients with primary fibromyalgia. J Rheumatol 31(2):364-378

10. Luerding R, Weigand T, Bogdahn U, Schmidt-Wilcke T (2008) Working memory performance is correlated with local brain morphology in the medial frontal and anterior cingulate cortex in fibromyalgia patients: Structural correlates of pain-cognition interaction. Brain 131(12):3222-3231. https://doi.org/10.1093/ brain/awn229

11. Giannotti E, Koutsikos K, Pigatto M, Rampudda ME, Doria A, Masiero S (2014) Medium-/long-term effects of a specific exercise protocol combined with patient education on spine mobility, chronic fatigue, pain, aerobic fitness and level of disability in fibromyalgia. Int Biomed Res. https://doi.org/10.1155/2014/47402 9

12. Staud R, Craggs JG, Perlstein WM, Robinson ME, Price DD (2008) Brain activity associated with slow temporal summation of C-fiber evoked pain in fibromyalgia patients and healthy controls. Eur J Pain 12(8):1078-1089. https://doi.org/10.1016/j.ejpai n.2008.02.002

13. Burgmer M et al (2009) Decreased gray matter volumes in the cingulo-frontal cortex and the amygdala in patients with fibromyalgia. Psychosom Med 71(5):566-573. https://doi.org/10.1097/ PSY.0b013e3181a32da0

14. Llinás RR, Ribary U, Jeanmonod D, Kronberg E, Mitra PP (1999) Thalamocortical dysrhythmia: a neurological and neuropsychiatric syndrome characterized by magnetoencephalography. Proc Natl Acad Sci USA 96(26):15222-15227. https://doi.org/10.1073/ pnas.96.26.15222 
15. Gücer G, Niedermeyer E, Long DM (1978) Thalamic EEG recordings in patients with chronic pain. J Neurol 219(1):47-61. https:// doi.org/10.1007/BF00313368

16. Fallon N, Chiu Y, Nurmikko T, Stancak A (2018) Altered theta oscillations in resting EEG of fibromyalgia syndrome patients. Eur J Pain (UK) 22(1):49-57. https://doi.org/10.1002/ejp.1076

17. Thut $\mathrm{G}$ et al (2017) Guiding transcranial brain stimulation by EEG/MEG to interact with ongoing brain activity and associated functions: a position paper. Clin Neurophysiol 128(5):843-857. https://doi.org/10.1016/j.clinph.2017.01.003

18. Del Felice A et al (2019) Personalized transcranial alternating current stimulation (tACS) and physical therapy to treat motor and cognitive symptoms in Parkinson's disease: a randomized crossover trial. NeuroImage Clin. 22:101768. https://doi.org/10.1016/j. nicl.2019.101768

19. Del Felice A, Magalini A, Masiero S (2015) Slow-oscillatory transcranial direct current stimulation modulates memory in temporal lobe epilepsy by altering sleep spindle generators: a possible rehabilitation tool. Brain Stimul 8(3):567-573. https://doi. org/10.1016/j.brs.2015.01.410

20. Reed T, Cohen Kadosh R (2018) Transcranial electrical stimulation (tES) mechanisms and its effects on cortical excitability and connectivity". J Inherit Metab Dis. 41(6):1123-1130. https://doi. org/10.1007/s10545-018-0181-4

21. Das S, Holland P, Frens MA, Donchin O (2016) Impact of transcranial direct current stimulation (tDCS) on neuronal functions. Front Neurosci. https://doi.org/10.3389/fnins.2016.00550

22. Passard A et al (2007) Effects of unilateral repetitive transcranial magnetic stimulation of the motor cortex on chronic widespread pain in fibromyalgia. Brain 130(10):2661-2670. https://doi. org/10.1093/brain/awm189

23. Bergmann TO, Groppa S, Seeger M, Mölle M, Marshall L, Siebner HR (2009) Acute changes in motor cortical excitability during slow oscillatory and constant anodal transcranial direct current stimulation. J Neurophysiol 102(4):2303-2311. https://doi. org/10.1152/jn.00437.2009

24. Marlow NM, Bonilha HS, Short EB (2013) Efficacy of transcranial direct current stimulation and repetitive transcranial magnetic stimulation for treating fibromyalgia syndrome: a systematic review. Pain Pract 13(2):131-145. https://doi.org/10.111 $1 / j .1533-2500.2012 .00562 . x$

25. Brighina F et al (2019) Brain modulation by electric currents in fibromyalgia: a structured review on non-invasive approach with transcranial electrical stimulation. Front Hum Neurosci 13:1-14. https://doi.org/10.3389/fnhum.2019.00040

26. Helfrich RF, Schneider TR, Rach S, Trautmann-Lengsfeld SA, Engel AK, Herrmann CS (2014) Entrainment of brain oscillations by transcranial alternating current stimulation. Curr Biol 24(3):333-339. https://doi.org/10.1016/j.cub.2013.12.041

27. McDonnell MD, Abbott D (2009) What is stochastic resonance? Definitions, misconceptions, debates, and its relevance to biology. PLoS Comput Biol. https://doi.org/10.1371/journal.pcbi.1000348

28. Dwan K, Li T, Altman DG, Elbourne D (2019) CONSORT 2010 statement: extension to randomised crossover trials. BMJ. https ://doi.org/10.1136/bmj.14378

29. Wolfe F et al (2016) Revisions to the 2010/2011 fibromyalgia diagnostic criteria. Semin Arthritis Rheum 46:319-329. https:// doi.org/10.1016/j.semarthrit.2016.08.012

30. Formaggio E, Masiero S, Bosco A, Izzi F, Piccione F, Del Felice A (2017) Quantitative EEG evaluation during robot-assisted foot movement. IEEE Trans Neural Syst Rehabil Eng 25(9):16331640. https://doi.org/10.1109/TNSRE.2016.2627058

31. Delorme A, Makeig S (2004) EEGLAB: An open source toolbox for analysis of single-trial EEG dynamics including independent component analysis. J Neurosci Methods 134(1):9-21. https://doi. org/10.1016/j.jneumeth.2003.10.009
32. Duffy FH, Bartels PH, Burchfiel JL (1981) Significance probability mapping: an aid in the topographic analysis of brain electrical activity. Electroencephalogr Clin Neurophysiol 51(5):455-462. https://doi.org/10.1016/0013-4694(81)90221-2

33. Gift AC (1989) Visual analogue scales: measurement of subjective phenomena. Nurs Res 38(5):286-288. https://doi. org/10.1097/00006199-198909000-00006

34. Ware JE, Sherbourne CD (1992) The MOS 36-item short-form health survey (Sf-36): I. conceptual framework and item selection. Med Care 30(6):473-483. https://doi.org/10.1097/00005 650-199206000-00002

35. Dozois DJA (2010) Beck depression inventory-II. In: The Corsini encyclopedia of psychology. Wiley

36. Derogatis LR (1983) The brief symptom inventory: an introductory report. Psychol Med 13(3):595-605. https://doi.org/10.1017/ S0033291700048017

37. Spielberger CD (1966) Theory and research on anxiety. In: Anxiety and behavior, pp 3-20

38. Frank L et al (2006) Validation of a new symptom impact questionnaire for mild to moderate cognitive impairment. Int 18(1):135-149. https://doi.org/10.1017/S1041610205002887

39. Royle J, Lincoln NB (2008) The everyday memory questionnaire-revised: development of a 13-item scale. Disabil Rehabil 30(2):114-121. https://doi.org/10.1080/09638280701223876

40. Santangelo $\mathrm{G}$ et al (2015) Normative data for the montreal cognitive assessment in an italian population sample. Neurol Sci 36(4):585-591. https://doi.org/10.1007/s10072-014-1995-y

41. Caffarra P, Vezzadini G, Dieci F, Zonato F, Venneri A (2002) ReyOsterrieth complex figure: normative values in an Italian population sample. Neurol Sci 22(6):443-447. https://doi.org/10.1007/ s100720200003

42. Nelson JM, Canivez GL, Watkins MW (2013) Structural and incremental validity of the wechsler adult intelligence scale-fourth edition with a clinical sample. Psychol Assess 25(2):618-630. https://doi.org/10.1037/a0032086

43. Brandt J (1991) The hopkins verbal learning test: development of a new memory test with six equivalent forms. Clin Neuropsychol 5(2):125-142. https://doi.org/10.1080/13854049108403297

44. Reitan RM (1958) Validity of the trail making test as an indicator of organic brain damage. Percept Mot Skills 8(3):271-276. https ://doi.org/10.2466/pms.1958.8.3.271

45. Newcombe F (1969) Missile wounds of the brain: a study of psychological deficits. Oxford University Press

46. Macfarlane GJ et al (2017) EULAR revised recommendations for the management of fibromyalgia. Ann Rheum Dis 76(2):318-328. https://doi.org/10.1136/annrheumdis-2016-209724

47. R Core Team (2018) R: a language and environment for statistical computing. R Foundation for Statistical Computing, Vienna. https ://www.R-project.org/

48. Thut G, Miniussi C (2009) New insights into rhythmic brain activity from TMS-EEG studies. Trends Cogn Sci 13(4):182-189. https://doi.org/10.1016/j.tics.2009.01.004

49. Cagnie B, Coppieters I, Denecker S, Six J, Danneels L, Meeus M (2014) Central sensitization in fibromyalgia? A systematic review on structural and functional brain MRI. Semin Arthritis Rheum 44(1):68-75. https://doi.org/10.1016/j.semarthrit.2014.01.001

50. Legrain V, Iannetti GD, Plaghki L, Mouraux A (2011) The pain matrix reloaded: a salience detection system for the body. Prog Neurobiol 93(1):111-124. https://doi.org/10.1016/j.pneur obio.2010.10.005

51. Castillo Saavedra L, Mendonca M, Fregni F (2014) Role of the primary motor cortex in the maintenance and treatment of pain in fibromyalgia. Med. Hypotheses 83(3):332-336. https://doi. org/10.1016/j.mehy.2014.06.007

52. Del Felice A, Daloli V, Masiero S, Manganotti P (2016) Contralesional cathodal versus dual transcranial direct current stimulation 
for decreasing upper limb spasticity in chronic stroke individuals: a clinical and neurophysiological study. J Stroke Cerebrovasc Dis 25(12):2932-2941. https://doi.org/10.1016/j.jstrokecerebrovasdis .2016 .08 .008

53. Jamil A, Yavari F, Kuo M-F, Nitsche MA (2020) NIBS as a research tool in clinical and translational neuroscience. In: Dell'Osso B, Di Lorenzo G (eds) Non invasive brain stimulation in psychiatry and clinical neurosciences. Springer, Cham, pp 43-59

54. Ströhle A (2020) Physical activity, exercise, depression and anxiety disorders. https://doi.org/10.1007/s00702-008-0092-X.

55. Wang HX et al (2020) Protocol on transcranial alternating current stimulation for the treatment of major depressive disorder: a randomized controlled trial. Chin Med J (Engl) 133(1):61-67. https://doi.org/10.1097/CM9.0000000000000589

56. Trammell JP, MacRae PG, Davis G, Bergstedt D, Anderson AE (2017) The relationship of cognitive performance and the thetaalpha power ratio is age-dependent: an eeg study of short term memory and reasoning during task and resting-state in healthy young and old adults. Front. Aging Neurosci 9:364. https://doi. org/10.3389/fnagi.2017.00364

57. Clark CR et al (2004) Spontaneous alpha peak frequency predicts working memory performance across the age span. Int J Psychophysiol 53(1):1-9. https://doi.org/10.1016/j.ijpsycho.2003.12.011
58. Mandolesi L et al (2018) Effects of physical exercise on cognitive functioning and wellbeing: biological and psychological benefits. Front Psychol. https://doi.org/10.3389/fpsyg.2018.00509

59. Bellato E et al (2012) Fibromyalgia syndrome: etiology, pathogenesis, diagnosis, and treatment. Treat Pain Res. https://doi. org/10.1155/2012/426130

60. McLaren ME, Nissim NR, Woods AJ (2018) The effects of medication use in transcranial direct current stimulation: a brief review. Brain Stimul 11(1):52-58. https://doi.org/10.1016/j. brs.2017.10.006

61. Nasreddine ZS et al (2005) The montreal cognitive assessment, MoCA: a brief screening tool for mild cognitive impairment. J Am Geriatr Soc 53(4):695-699. https://doi.org/10.111 1/j.1532-5415.2005.53221.x

62. Hofheimer JA (2016) Neuropsychological assessment. In: The curated reference collection in neuroscience and biobehavioral psychology, pp 425-438

63. LoSasso GL, Rapport LJ, Axelrod BN, Reeder KP (1998) Intermanual and alternate-form equivalence on the trail making tests. J Clin Exp Neuropsychol 20(1):107-110. https://doi.org/10.1076/ jcen.20.1.107.1487 